\title{
Pacific
}

Journal of

Mathematics

\section{BOURGAIN ALGEBRAS ON THE UNIT DISK}

Joseph A. Cima, Karel M. Stroethoff and Keith Yale 


\title{
BOURGAIN ALGEBRAS ON THE UNIT DISK
}

\author{
Joseph A. Cima, Karel Stroethoff and Keith Yale
}

The Bourgain algebra of $H^{\infty}(\mathbb{D})$ relative to $L^{\infty}(\mathbb{D})$ is shown to be $H^{\infty}(\mathbb{D})+C(\overline{\mathbb{D}})+V$, where $V$ is an ideal of functions in $L^{\infty}(\mathbb{D})$ which vanish in an appropriate sense near the boundary of $\mathbb{D}$.

1. Introduction. Let $\mathscr{X}$ be a commutative Banach algebra with an identity and let $\mathscr{A}$ be a linear subspace of $\mathscr{X}$. J. Cima and R. Timoney [6] introduced the notion of the Bourgain algebra based on ideas of J. Bourgain [3]: the Bourgain algebra $\mathscr{A}_{b}$ consists of those $f$ in $\mathscr{X}$ such that

$$
\text { if } f_{n} \rightarrow 0 \text { weakly in } \mathscr{A} \text {, then } \operatorname{dist}\left(f_{n} f, \mathscr{A}\right) \rightarrow 0 \text {. }
$$

The distance $\operatorname{dist}\left(f_{n} f, \mathscr{A}\right)$ between $f_{n} f$ and $\mathscr{A}$ is the quotient norm of the coset $f_{n} f+\mathscr{A}$ in the space $\mathscr{X} / \mathscr{A}$. The proof in [6] shows that $\mathscr{A}_{b}$ is a closed subalgebra of $\mathscr{X}$ and if $\mathscr{A}$ is an algebra then $\mathscr{A} \subseteq \mathscr{A}_{b}$. It is important to note that $\mathscr{A}_{b}$ depends upon the space $\mathscr{X}$ even though this is not reflected in the notation. For a brief survey of Bourgain algebras see K. Yale [16].

Let $H^{\infty}(\mathbb{D})$ be the algebra of bounded analytic functions on the open unit disk $\mathbb{D}$. There are at least three different natural spaces $\mathscr{X}$ containing $H^{\infty}(\mathbb{D})$. First we can let $\mathbb{T}=\partial \mathbb{D}=\{z \in \mathbb{C}:|z|=1\}$ be the unit circle and consider the algebra $H^{\infty}(\mathbb{T})$ of boundary values of $H^{\infty}(\mathbb{D})$ functions as a subalgebra of $\mathscr{X}=L^{\infty}(\mathbb{T})$. In this context, J. Cima, S. Janson and K. Yale [5] showed that $H^{\infty}(\mathbb{T})_{b}=$ $H^{\infty}(\mathbb{T})+C(\mathbb{T})$. Another setting is to use the Gelfand map and regard $H^{\infty}(\mathbb{D})$ as a subalgebra of $\mathscr{X}=C(\mathscr{M})$, where $\mathscr{M}$ denotes the maximal ideal space of $H^{\infty}(\mathbb{D})$. In this context $H^{\infty}(\mathbb{D})_{b}$ has been determined by $P$. Ghatage, S. Sun and D. Zheng [10]. Yet another natural setting is to regard $H^{\infty}(\mathbb{D})$ as a subalgebra of $\mathscr{X}=L^{\infty}(\mathbb{D})$, where $L^{\infty}(\mathbb{D})$ is the usual space of equivalence classes of essentially bounded measurable functions on $\mathbb{D}$ with respect to area measure. The purpose of this paper is to determine $H^{\infty}(\mathbb{D})_{b}$ in the latter context. There is no Chang-Marshall theory for $L^{\infty}(\mathbb{D})$ in contrast to the well-known description of subalgebras between $H^{\infty}(\mathbb{T})$ and $L^{\infty}(\mathbb{T})$ which was used in [5] to determine $H^{\infty}(\mathbb{T})_{b}$. For a survey of the 
Douglas algebra problem for $L^{\infty}(\mathbb{D})$, i.e., the problem of the determination of the algebras between $H^{\infty}(\mathbb{D})$ and $L^{\infty}(\mathbb{D})$, see P. Gorkin [11]. Ideas arising from the study of Bourgain algebras may shed some light on the Douglas algebra problem. K. Izuchi [13] has recently developed an abstract approach to the problem of determining Bourgain algebras in a variety of settings, which do not encompass our methods and results.

A substantial study of Bourgain algebras of Douglas subalgebras of $L^{\infty}(\mathbb{T})$ is contained in [12], while a variety of interesting results concerning Bourgain algebras of some special subalgebras of $C(\mathscr{M})$ are found in [17].

We show in $\S 4$ that $H^{\infty}(\mathbb{D})_{b}=H^{\infty}(\mathbb{D})+C(\overline{\mathbb{D}})+V$, where $V$ is an ideal of functions in $L^{\infty}(\mathbb{D})$ which vanish in an appropriate sense near the boundary of $\mathbb{D}$. Section 2 contains preliminaries as well as several examples. Functions in $H^{\infty}(\mathbb{D})_{b}$ have "boundary values" and this is proved in $\S 3$. The boundary value result enables one to reduce the determination of $H^{\infty}(\mathbb{D})_{b}$ to the known result for $H^{\infty}(\mathbb{T})_{b}$. In $\S 5$ we investigate the connection between $H^{\infty}(\mathbb{D})_{b}$ and the algebra $A Q$ of bounded symbols of compact Hankel operators on the Bergman space. Let $A(\mathbb{D})$ denote the disk algebra, the algebra of continuous functions on $\overline{\mathbb{D}}$ which are analytic on $\mathbb{D}$. In $\S 6$ we study the boundary behavior of analytic functions in the Bourgain algebras $A(\mathbb{D})_{b}$ relative to $L^{\infty}(\mathbb{D})$. We end the paper with further remarks and questions in $\S 7$.

We wish to thank K. Izuchi and R. Mortini for their many helpful discussions concerning this work. We are especially grateful to the referee for pointing out a major error in an earlier version of this paper and for suggesting a better viewpoint.

2. Preliminaries. Several observations concerning weakly null sequences in $H^{\infty}(\mathbb{D})$ will be helpful in the determination of the Bourgain algebra $H^{\infty}(\mathbb{D})_{b}$. The mapping $f \mapsto f^{*}$, where $f^{*}(\zeta)=$ $\lim _{r \rightarrow 1^{-}} f(r \zeta)$, for almost every $\zeta \in \mathbb{T}$, provides an isometric isomorphism from $H^{\infty}(\mathbb{D})$ onto $H^{\infty}(\mathbb{T})$. Thus $f_{n} \rightarrow 0$ weakly in $H^{\infty}(\mathbb{D})$ if and only if $f_{n}^{*} \rightarrow 0$ weakly in $H^{\infty}(\mathbb{T})$. If $f_{n} \rightarrow 0$ weakly in $H^{\infty}(\mathbb{D})$ then $\left\{f_{n}\right\}$ is uniformly bounded $\left(\left\|f_{n}\right\|_{\infty} \leq M<\infty\right.$ for all $\left.n \geq 1\right)$ and $f_{n} \rightarrow 0$ uniformly on compact subsets of $\mathbb{D}$.

Any sequence in $H^{\infty}(\mathbb{D})$ which is uniformly bounded and converges to 0 uniformly on compact subsets of $\mathbb{D}$ will be called normal null. Any weakly null sequence is certainly normal null, but not conversely. 
For example, $f_{n}(z)=z^{n}$ is normal null but does not converge to 0 weakly.

For a subalgebra $\mathscr{A} \subseteq L^{\infty}(\mathbb{D})$ we define the normal null algebra $\mathscr{A}_{N}$ to be the set of those functions $f$ in $L^{\infty}(\mathbb{D})$ such that

$$
\text { if }\left\{f_{n}\right\} \text { is normal null in } \mathscr{A} \text {, then } \operatorname{dist}\left(f_{n} f, \mathscr{A}\right) \rightarrow 0 \text {. }
$$

A minor modification of the argument in [6] shows that $\mathscr{A}_{N}$ is a closed subalgebra of $L^{\infty}(\mathbb{D})$ with $\mathscr{A} \subseteq \mathscr{A}_{N}$. Clearly $H^{\infty}(\mathbb{D})_{N} \subseteq H^{\infty}(\mathbb{D})_{b}$ since every weakly null sequence in $H^{\infty}(\mathbb{D})$ is normal null. For a closed subalgebra $\mathscr{A} \subseteq L^{\infty}(\mathbb{D})$ and $f \in L^{\infty}(\mathbb{D})$ define the Hankel type operator $S_{f}: \mathscr{A} \rightarrow L^{\infty}(\mathbb{D}) / \mathscr{A}$ by $S_{f} h=f h+\mathscr{A}, h \in \mathscr{A}$. Note that the Bourgain algebra $\mathscr{A}_{b}$ consists of those $f$ in $L^{\infty}(\mathbb{D})$ for which the operator $S_{f}$ is completely continuous. The set $\mathscr{A}_{w c}$ of functions $f$ in $L^{\infty}(\mathbb{D})$ for which $S_{f}$ is weakly compact is also a closed subalgebra of $L^{\infty}(\mathbb{D})$ with $\mathscr{A} \subseteq \mathscr{A}_{w c}$ as is shown by the duality argument of B. Cole and T. Gamelin [7], Lemma 4.2. Note that $f \in \mathscr{A}_{w c}$ in case the operator $S_{f}: \mathscr{A} \rightarrow L^{\infty}(\mathbb{D}) / \mathscr{A}$ defined by $S_{f} h=f h+\mathscr{A}, h \in \mathscr{A}$, is weakly compact while $f \in \mathscr{A}_{b}$ in case the operator $S_{f}$ is completely continuous. Now weakly compact operators are completely continuous in any space which has the Dunford-Pettis property and so $H^{\infty}(\mathbb{D})_{w c} \subseteq H^{\infty}(\mathbb{D})_{b}$ follows immediately from the remarkable theorem of J. Bourgain [2] that $H^{\infty}(\mathbb{D})$ has the DunfordPettis property. If $f \in H^{\infty}(\mathbb{D})_{N}$ then a normal families argument shows that $S_{f}$ is compact and hence weakly compact. In particular, we have the inclusion $H^{\infty}(\mathbb{D})_{N} \subseteq H^{\infty}(\mathbb{D})_{w c}$.

The space $H^{\infty}(\mathbb{D})$ is well supplied with weakly null sequences which do not converge to zero in norm. Such sequences arise from peak point and from interpolation type constructions; the following lemma is useful in this respect.

LEMMA 1. Suppose that $\left\{f_{n}\right\}$ is a sequence of functions in $H^{\infty}(\mathbb{D})$ and $M$ is a constant such that

$$
\sum_{n=1}^{\infty}\left|f_{n}(z)\right| \leq M, \quad z \in \mathbb{D} .
$$

Then $f_{n} \rightarrow 0$ weakly in $H^{\infty}(\mathbb{D})$.

Proof. See [5].

If $\left\{z_{n}\right\}$ is an interpolating sequence in the unit disk, by the $P$. Beurling Interpolation Theorem (see [9]) there exist functions $f_{n}$ in 
$H^{\infty}(\mathbb{D})$ such that $f_{n}\left(z_{m}\right)=\delta_{n m}$ and for which (3) holds. Consequently, $f_{n} \rightarrow 0$ weakly in $H^{\infty}(\mathbb{D})$.

Let $\left\{\zeta_{n}\right\}$ be a sequence of distinct points on the circle $\mathbb{T}$ which converges to a point $\zeta$ which is not equal to any of the $\zeta_{n}$. Since each point of $\mathbb{T}$ is a peak point for the disk algebra $A(\mathbb{D})$, there exist functions $g_{n} \in A(\mathbb{D})$ such that $g_{n}\left(\zeta_{n}\right)=1$ and $\left|g_{n}(z)\right|<1$ if $z \in \overline{\mathbb{D}}$, but $z \neq \zeta_{n}$. Construct a disjoint collection $\left\{\mathscr{O}_{n}: n \geq 1\right\}$ of open subsets of $\mathbb{T}$ such that $\zeta_{n} \in \mathscr{O}_{n}$ and $\sup \left\{\left|g_{n}(\zeta)\right|: \zeta \in \mathbb{T} \backslash \mathscr{O}_{n}\right\}<1$, and for each $n$ choose a positive integer $k_{n}$ so large that $f_{n}(z)=g_{n}(z)^{k_{n}}$ is small on $\mathbb{T} \backslash \mathscr{O}_{n}:\left|f_{n}(\zeta)\right| \leq 1 / 2^{n}$ for $\zeta \in \mathbb{T} \backslash \mathscr{O}_{n}$. Hence (3) holds with $M=2$ and we conclude that $f_{n} \rightarrow 0$ weakly in $H^{\infty}(\mathbb{T})$ and hence in $H^{\infty}(\mathbb{D})$. Since the sequence $\left\{f_{n}\right\}$ is in $A(\mathbb{D})$ it is also weakly null in $A(\mathbb{D})$. Clearly $f_{n}\left(\zeta_{n}\right)=1$ for all $n \geq 1$. A concrete example is given by

$$
f_{n}(z)=\left(\frac{z+\zeta_{n}}{2}\right)^{k_{n}}
$$

A few simple examples and a proposition will serve to fix the ideas and to motivate the main theorems.

EXAMPLE 2. Let $g$ be an arbitrary function in $L^{\infty}(\mathbb{D}), K \subset \mathbb{D}$ compact and put $f=\chi_{K} g$ where $\chi_{K}$ is the characteristic function of $K$. Then $f \in H^{\infty}(\mathbb{D})_{N}$ because $\operatorname{dist}\left(f_{n} f, H^{\infty}(\mathbb{D})\right) \leq\left\|f_{n} \chi_{K} g-0\right\|_{\infty} \leq$ $\|g\|_{\infty}\left\|f_{n} \chi_{K}\right\|_{\infty} \rightarrow 0$ for any normal null sequence $\left\{f_{n}\right\}$ in $H^{\infty}(\mathbb{D})$.

\section{Proposition 3. $H^{\infty}(\mathbb{D})+C(\overline{\mathbb{D}}) \subseteq H^{\infty}(\mathbb{D})_{N}$.}

Proof. By the Stone-Weierstrass Theorem it suffices to show that the function $\bar{z}$ belongs to $H^{\infty}(\mathbb{D})_{N}$. Let $\left\{f_{n}\right\}$ be a normal null sequence in $H^{\infty}(\mathbb{D})$, and for each positive integer $n$ let $h_{n} \in H^{\infty}(\mathbb{D})$ be defined by

$$
h_{n}(z)=\frac{f_{n}(z)-f_{n}(0)}{z}, \quad \text { for } z \in \mathbb{D} \backslash\{0\} .
$$

It is easily seen that the sequence $\left\{h_{n}\right\}$ is normal null in $H^{\infty}(\mathbb{D})$. Let $M$ be a finite positive number such that $\left\|h_{n}\right\|_{\infty} \leq M$ for each $n \geq 1$. Using $\bar{z} f_{n}(z)=|z|^{2} h_{n}(z)+\bar{z} f_{n}(0)$ we see that

$$
\begin{aligned}
\operatorname{dist}\left(\bar{z} f_{n}, H^{\infty}(\mathbb{D})\right) \leq & \left\|\bar{z} f_{n}-h_{n}\right\|_{\infty} \leq\left(1-r^{2}\right) M \\
& +\max _{|z| \leq r}\left|h_{n}(z)\right|+\left|f_{n}(0)\right|,
\end{aligned}
$$

for each $0<r<1$. Taking the limit superior as $n \rightarrow \infty$, and subsequently letting $r \rightarrow 1^{-}$, it follows that $\operatorname{dist}\left(\bar{z} f_{n}, H^{\infty}(\mathbb{D})\right) \rightarrow 0$ as $n \rightarrow \infty$, proving that the function $\bar{z}$ belongs to $H^{\infty}(\mathbb{D})_{N}$. 
It follows from Example 2 and Proposition 3 that $H^{\infty}(\mathbb{D})_{N}$, and thus $H^{\infty}(\mathbb{D})_{b}$, contains all functions on the unit disk which are " $H^{\infty}(\mathbb{D})+C(\overline{\mathbb{D}})$ near the boundary," that is, coincide with a function from the algebra $H^{\infty}(\mathbb{D})+C(\overline{\mathbb{D}})$ on the complement of a compact subset of $\mathbb{D}$. We will actually prove that $H^{\infty}(\mathbb{D})_{b}$ is the sum of $H^{\infty}(\mathbb{D})+C(\overline{\mathbb{D}})$ and (equivalence classes of) functions that "vanish near the boundary." This statement will be made precise in Theorem 12 of $\S 4$.

EXAMPLE 4. Let $U=\{z \in \mathbb{D}: \operatorname{Im} z>0\}$ be the upper open half disk and let $f=\chi_{U}$. Then $f \notin H^{\infty}(\mathbb{D})_{b}$. For if we suppose that $f \in H^{\infty}(\mathbb{D})_{b}$, then for every weakly null sequence $\left\{f_{n}\right\}$ in $H^{\infty}(\mathbb{D})$ there exists a sequence $\left\{g_{n}\right\}$ in $H^{\infty}(\mathbb{D})$, such that $\left\|f_{n} f-g_{n}\right\|_{\infty} \rightarrow 0$ as $n \rightarrow \infty$. Then $f_{n}-g_{n} \rightarrow 0$ uniformly on the closure of $U$ relative to $\mathbb{D}$ while $g_{n} \rightarrow 0$ uniformly on the closure of $\mathbb{D} \backslash U$ relative to $\mathbb{D}$ since $f_{n} f-g_{n}=-g_{n}$ on $\mathbb{D} \backslash U$. Hence $f_{n}=\left(f_{n}-g_{n}\right)+g_{n} \rightarrow 0$ uniformly on the line segment $(-1,1)=\bar{U} \cap \overline{\mathbb{D} \backslash U} \cap \mathbb{D}$. This is a contradiction because there are many weakly null sequences which do not converge uniformly to zero on the line segment $(-1,1)$; in particular, if $\left\{z_{n}\right\}$ is an interpolating sequence in $(-1,1)$ the $\mathrm{P}$. Beurling sequence $\left\{f_{n}\right\}$ does not converge uniformly to zero on $(-1,1)$ since $f_{n}\left(z_{n}\right)=1$ for each $n \geq 1$.

EXAMPLE 5. Let $\left\{r_{n}\right\}$ be a sequence of positive numbers increasing to 1 and let $S_{n}=\left\{z \in \mathbb{C}: r_{2 n-1}<|z|<r_{2 n}\right\}$. The values of the function $f=\chi_{S}$, where $S=\bigcup_{n=1}^{\infty} S_{n}$, oscillate as $|z| \rightarrow 1^{-}$, since $S$ is the union of concentric annuli. Thus the radial limit, $f^{*}(\zeta)$, of $f$ does not exist for any $\zeta \in \mathbb{T}$. The argument given in the previous example can be used to show $f \notin H^{\infty}(\mathbb{D})_{b}$. (The arbitrary weakly null sequence $\left\{f_{n}\right\}$ is forced to converge uniformly to 0 on the union of the circles $\bigcup_{n=1}^{\infty}\left\{z \in \mathbb{C}:|z|=r_{n}\right\}=\bar{S} \cap \overline{\mathbb{D} \backslash S}$ so that by the maximum modulus principle $\left\|f_{n}\right\|_{\infty} \rightarrow 0$.)

These examples are typical. Thus $f$ will belong to $H^{\infty}(\mathbb{D})_{b}$ if it vanishes near the boundary but the existence of boundary values in the absence of interior regularity is not sufficient to make $f$ belong to $H^{\infty}(\mathbb{D})_{b}$. Bad boundary behavior of the function $f$ will always force $f \notin H^{\infty}(\mathbb{D})_{b}$ as in Example 5. These statements are given precise form by the main theorems of $\S \S 3$ and 4 . The proofs of the main theorems require more delicate properties of weakly null sequences than were used in the examples.

Since $L^{\infty}(\mathbb{D})$ consists of equivalence classes of essentially bounded 
measurable functions, simple pointwise definitions of boundary values do not make sense because one can always modify a function on a set of area measure zero so that its limit does not exist as $|z| \rightarrow 1^{-}$. However, technically useful definitions can be made for essential nontangential limits and oscillations and these notions have an important role in the proofs of our principal theorems.

For $\zeta \in \mathbb{T}$ and $0<R<1$ the open non-tangential cone $\Gamma_{R}(\zeta)$ at $\zeta$ is the interior of the convex hull of $\zeta$ and the disk $\{z \in \mathbb{C}:|z| \leq R\}$. We say that $f$ has essential non-tangential limit $L$ at $\zeta$ if

$$
\text { ess } \sup \left\{|f(z)-L|: z \in \Gamma_{R}(\zeta),|z|>1-\delta\right\} \rightarrow 0 \quad \text { as } \delta \rightarrow 0^{+}
$$

for all $0<R<1$, in which case we will write $f^{*}(\zeta)$ for $L$. We define $B V$ to be the set of $f$ in $L^{\infty}(\mathbb{D})$ such that an essential non-tangential limit $f^{*}(\zeta)$ exists for almost every $\zeta \in \mathbb{T}$. In order to give another description of $B V$ we will introduce more notation. For $f \in L^{\infty}(\mathbb{D})$ and a nonempty set $E \subset \mathbb{D}$ define the essential oscillation of $f$ over $E$ to be

$$
\omega(f, E)=\operatorname{ess} \sup \{|f(z)-f(w)|: z, w \in E\} .
$$

For each $z \in \overline{\mathbb{D}}$ and $0<\delta<1$ we will write $E(z, \delta)=\{w \in$ $\mathbb{D}:|w-z|<\delta\}$. For $f \in L^{\infty}(\mathbb{D})$ define the essential oscillation of $f$ at $z \in \overline{\mathbb{D}}$ to be

$$
\omega(f, z)=\lim _{\delta \rightarrow 0^{+}} \omega(f, E(z, \delta)) .
$$

If we define the essential non-tangential oscillation of $f$ at $\zeta \in \mathbb{T}$ by

$$
\omega_{R}(f, \zeta)=\lim _{\delta \rightarrow 0^{+}} \omega\left(f, E(\zeta, \delta) \cap \Gamma_{R}(\zeta)\right),
$$

then we have the following description of $B V$.

Proposition 6.

$$
\begin{aligned}
B V=\left\{f \in L^{\infty}(\mathbb{D}):\right. & \omega_{R}(f, \zeta)=0 \\
& \text { for all } 0<R<1 \text { and almost every } \zeta \in \mathbb{T}\} .
\end{aligned}
$$

Proof. If for $\zeta \in \mathbb{T}$ the essential non-tangential limit $f^{*}(\zeta)$ exists, then a simple application of the triangle inequality shows that $\omega_{R}(f, \zeta)=0$. Conversely, if $\omega_{R}(f, \zeta)=0$, for all $0<R<1$, then it is possible to choose a suitable sequence $\left\{z_{n}(R)\right\}$ in $\mathbb{D}$, converging to $\zeta$, for which $\left\{f\left(z_{n}(R)\right)\right\}$ is Cauchy with limit, $f^{*}(\zeta)$, independent of $R$. A suitable sequence can be constructed by taking $z_{n}(R) \in$ $E(\zeta, 1 / n) \cap \Gamma_{R}(\zeta)=S$ for which ess $\sup \left\{\left|f(z)-f\left(z_{n}(R)\right)\right|: z \in S\right\} \leq$ $\omega(f, S)$. The details are left to the reader. 
Note that if $f, g \in L^{\infty}(\mathbb{D})$, and $f$ has essential limit $f^{*}(\zeta)$ as $z$ approaches $\zeta \in \mathbb{T}$ in the cone $\Gamma_{R}(\zeta)$, then

$$
\omega_{R}(f g, \zeta)=\left|f^{*}(\zeta)\right| \omega_{R}(g, \zeta) \text {. }
$$

Also note that if $\omega_{R}(g, \zeta)=0$, then $\omega_{R}(f, \zeta)=\omega_{R}(f-g, \zeta)$.

Since functions in $H^{\infty}(\mathbb{D})$ have non-tangential limits almost everywhere on the circle we have $H^{\infty}(\mathbb{D}) \subseteq B V$. From the main theorem in $\S 3$ it will follow that $B V$ is closed and $(B V)_{b}=B V$.

3. Boundary Value Theorem. As we emphasized in $\S 2$ the algebra $H^{\infty}(\mathbb{D})_{b}$ is a subset of the Banach algebra $L^{\infty}(\mathbb{D})$ and as such its members are equivalence classes of functions. The following lemma on metric density is an important tool in the proof of the theorem of this section. Although it is valid in more general situations, we state it for the case of subsets of the real numbers $\mathbb{R}^{1}$. If $E$ is a Lebesgue measurable subset of $\mathbb{R}^{1}$ the quantity

$$
D(E, x) \equiv \lim _{\delta \rightarrow 0^{+}} \frac{|E \cap(x-\delta, x+\delta)|}{2 \delta},
$$

if it exists, is the metric density of $E$ at the point $x$. It is well known that $D(E, x)=1$ for almost all $x$ in $E$. Let $D(E)=$ $\{x \in E: D(E, x)=1\}$, so that $|D(E)|=|E|$, and denote $J_{n}(x)=$ $\left(x-\frac{1}{n}, x+\frac{1}{n}\right)$. We identify $y \in J_{n}(x)$ with $e^{i y}$ when working on the circle $\mathbb{T}$.

LEMMA 7. Let $E$ and $Z$ be measurable subsets of the circle $\mathbb{T}$ with $|E|>0$ and $|Z|=0$. Then each $\zeta \in D(E)$ is the limit of a sequence $\left\{\zeta_{n}\right\}$ of distinct points in $D(E) \backslash Z$.

Proof. Left to the reader.

THEOREM 8. $H^{\infty}(\mathbb{D})_{b} \subseteq B V$.

Proof. Assume not and choose an $f$ in $H^{\infty}(\mathbb{D})_{b}$ whose non-tangential limits fail to exist on a set $B \subseteq \mathbb{T}$ with $|B|>0$. For each $\zeta \in B$ there is an $R(\zeta)$ in $(0,1)$ such that $\omega_{R(\zeta)}(f, \zeta) \geq \delta(\zeta)>0$. Setting $B_{j k}=\left\{\zeta \in B: \delta(\zeta) \geq \frac{1}{j}\right.$ and $\left.R(\zeta) \leq 1-\frac{1}{k}\right\}$ for $j, k \in \mathbb{Z}^{+}$, we have $B=\bigcup\left\{B_{j k}: j, k \in \mathbb{Z}^{+}\right\}$. Since $|B|>0$ there are $j$ and $k$ in $\mathbb{Z}^{+}$for which $\left|B_{j k}\right|>0$. We replace $B$ with the set $B_{j k}$ and henceforth we have

$$
\omega_{R}(f, \zeta) \geq \delta>0
$$

for all $\zeta \in B$, where $0<R<1$. 
For $\zeta$ fixed in $D(B)$ we apply the preceding lemma to obtain a sequence $\left\{\zeta_{n}\right\}$ in $D(B)$ which converges to $\zeta$. Let $\left\{f_{n}\right\}$ in $A(\mathbb{D})$ be a weakly null sequence of peak point functions associated with the sequence $\left\{\zeta_{n}\right\}$. Since $f \in H^{\infty}(\mathbb{D})_{b}$ there is a sequence $\left\{h_{n}\right\}$ in $H^{\infty}(\mathbb{D})$ such that $\left\|f f_{n}-h_{n}\right\|_{\infty}=\varepsilon_{n} \rightarrow 0$ as $n \rightarrow \infty$. Then for almost every $\xi \in \mathbb{T}$,

$$
\left|f_{n}(\xi)\right| \delta \leq\left|f_{n}(\xi)\right| \omega_{R}(f, \xi)=\omega_{R}\left(f f_{n}, \xi\right)=\omega_{R}\left(f f_{n}-h_{n}, \xi\right) \leq 2 \varepsilon_{n} .
$$

Since we here may choose $\xi \in B$ so close to $\zeta_{n}$ that $\left|f_{n}(\xi)\right|>1 / 2$, the above inequality yields $\delta<4 \varepsilon_{n}$, a contradiction.

REMARK 9. In the above proof only two properties of $H^{\infty}(\mathbb{D})$ were used. First, functions in $H^{\infty}(\mathbb{D})$ have non-tangential limits almost everywhere on the unit circle, i.e., $H^{\infty}(\mathbb{D}) \subseteq B V$. Secondly, $H^{\infty}(\mathbb{D})$ contains the particular weakly null sequence of peak point functions $\left\{f_{n}\right\}$ simply because $f_{n} \in A(\mathbb{D}) \subseteq H^{\infty}(\mathbb{D})$. Thus the proof just given actually shows more:

If $\mathscr{A}$ is a linear subspace of $L^{\infty}(\mathbb{D})$ which satisfies $A(\mathbb{D}) \subseteq \mathscr{A} \subseteq$ $B V$, then $\mathscr{A}_{b} \subseteq B V$.

As an immediate consequence we have $B V_{b}=B V$.

The following theorem generalizes Example 4 of the previous section.

THEOREM 10. If $f \in H^{\infty}(\mathbb{D})_{b}$, then $\omega(f, z) \rightarrow 0$ as $|z| \rightarrow 1^{-}$.

Proof. Let $f \in H^{\infty}(\mathbb{D})_{b}$ and assume that $\left\{z_{n}\right\}$ in $\mathbb{D}$ with $\left|z_{n}\right| \rightarrow$ $1^{-}$. By going to a subsequence, which we will not relabel, we can furthermore assume that the sequence $\left\{z_{n}\right\}$ is interpolating. Choose a weakly null sequence $\left\{f_{n}\right\}$ of interpolating functions in $H^{\infty}(\mathbb{D})$ with $f_{n}\left(z_{m}\right)=\delta_{n m}$. Since $f \in H^{\infty}(\mathbb{D})_{b}$, there is a sequence $\left\{g_{n}\right\}$ in $H^{\infty}(\mathbb{D})$ such that $\left\|f f_{n}-g_{n}\right\|_{\infty} \rightarrow 0$ as $n \rightarrow \infty$. Then

$$
\begin{aligned}
\omega\left(f, z_{n}\right) & =\left|f_{n}\left(z_{n}\right)\right| \omega\left(f, z_{n}\right)=\omega\left(f f_{n}, z_{n}\right) \\
& =\omega\left(f f_{n}-g_{n}, z_{n}\right) \leq 2\left\|f f_{n}-g_{n}\right\|_{\infty},
\end{aligned}
$$

and so $\omega\left(f, z_{n}\right) \rightarrow 0$ as $n \rightarrow 0$.

REMARK 11. A careful analysis of the above proof shows that more generally we have the following result:

Let $\mathscr{A}$ be a linear subspace of $L^{\infty}(\mathbb{D})$ such that $H^{\infty}(\mathbb{D}) \subseteq \mathscr{A} \subseteq$ $C(\mathbb{D})$, and suppose that $f \in \mathscr{A}_{b}$. Then $\omega(f, z) \rightarrow 0$ as $|z| \rightarrow 1^{-}$.

4. The Structure Theorem. In this section we will give our description of the Bourgain algebra $H^{\infty}(\mathbb{D})_{b}$. 
THEOREM 12. $H^{\infty}(\mathbb{D})_{b}=H^{\infty}(\mathbb{D})_{w c}=H^{\infty}(\mathbb{D})_{N}=H^{\infty}(\mathbb{D})+C(\overline{\mathbb{D}})+V$, where $V=\left\{g \in L^{\infty}(\mathbb{D}):\left\|g \chi_{\mathbb{D} \backslash r \mathbb{D}}\right\|_{\infty} \rightarrow 0\right.$ as $\left.r \rightarrow 1^{-}\right\}$.

Proof. In Proposition 3 we have already shown that $H^{\infty}(\mathbb{D})+C(\overline{\mathbb{D}})$ $\subseteq H^{\infty}(\mathbb{D})_{N}$. Noting that $V$ is the closure in $L^{\infty}(\mathbb{D})$ of the set of all functions with compact support in $\mathbb{D}$, it follows from Example 2 and the fact that $H^{\infty}(\mathbb{D})_{N}$ is closed, that also $V \subseteq H^{\infty}(\mathbb{D})_{N}$. Thus we have $H^{\infty}(\mathbb{D})+C(\overline{\mathbb{D}})+V \subseteq H^{\infty}(\mathbb{D})_{N} \subseteq H^{\infty}(\mathbb{D})_{b}$.

Now let $f \in H^{\infty}(\mathbb{D})_{b}$. By the Boundary Value Theorem $f$ has non-tangential limits almost everywhere on $\mathbb{T}$. Let $f^{*}$ denote the limit function on $\mathbb{T}$. We will first argue that $f^{*} \in H^{\infty}(\mathbb{T})_{b}$. Let $\left\{\varphi_{n}\right\}$ be weakly null in $H^{\infty}(\mathbb{T})$, and for each positive integer $n$ choose $f_{n} \in H^{\infty}(\mathbb{D})$ such that $\varphi_{n}=f_{n}^{*}$. Because $f \in H^{\infty}(\mathbb{D})_{b}$ and $\left\{f_{n}\right\}$ is weakly null in $H^{\infty}(\mathbb{D})$, there exist $g_{n} \in H^{\infty}(\mathbb{D})$ such that $\varepsilon_{n}=$ $\left\|f_{n} f-g_{n}\right\|_{\infty} \rightarrow 0$ as $n \rightarrow \infty$. Since the mapping $F \mapsto F^{*}$ is a contractive homomorphism of $B V$ onto $L^{\infty}(\mathbb{T})$ we conclude that $\left\|f^{*} f_{n}^{*}-g_{n}^{*}\right\|_{L^{\infty}(\mathbb{T})} \leq \varepsilon_{n}$. This proves our claim that $f^{*} \in H^{\infty}(\mathbb{T})_{b}$.

Denoting the Poisson integral of a function $\varphi \in L^{\infty}(\mathbb{T})$ by $\mathscr{P}[\varphi]$, we now put $g=f-\mathscr{P}\left[f^{*}\right]$. By [5], $H^{\infty}(\mathbb{T})_{b}=H^{\infty}(\mathbb{T})+C(\mathbb{T})$, so $f^{*} \in H^{\infty}(\mathbb{T})+C(\mathbb{T})$. As $\mathscr{P}$ maps $H^{\infty}(\mathbb{T})+C(\mathbb{T})$ into $H^{\infty}(\mathbb{D})+$ $C(\overline{\mathbb{D}}), \mathscr{P}\left[f^{*}\right] \in H^{\infty}(\mathbb{D})+C(\overline{\mathbb{D}}) \subseteq H^{\infty}(\mathbb{D})_{b}$, and we conclude that $g \in H^{\infty}(\mathbb{D})_{b}$. We claim that actually $g \in V$.

Assuming that $g \notin V$, there exists a sequence $\left\{r_{n}\right\}$ in the interval $(0,1)$ tending to 1 and a positive number $\delta$ such that $\left\|g \chi_{\mathbb{D} \backslash r_{n} \mathbb{D}}\right\|_{\infty} \geq$ $\delta$, for all $n \in \mathbb{Z}^{+}$. Put $A_{n}=\left\{z \in \mathbb{D}:\left|g \chi_{\mathbb{D} \backslash r_{n} \mathbb{D}}(z)\right|>\delta / 2\right\}$. Then $\left|A_{n}\right|>0$. Let $z_{n}$ be a point of density of the set $A_{n}$. Noting that $A_{n} \subseteq \mathbb{D} \backslash r_{n} \mathbb{D}$ we see that $\left|z_{n}\right| \rightarrow 1$ as $n \rightarrow \infty$. By passing to a subsequence we may assume that $\left\{z_{n}\right\}$ is an interpolating sequence. Let $\left\{f_{n}\right\}$ be a sequence in $H^{\infty}(\mathbb{D})$ such that $f_{n}\left(z_{n}\right)=1$ and $f_{n} \rightarrow$ 0 weakly. For each $n \in \mathbb{Z}^{+}$, by the continuity of $f_{n}$ at $z_{n}$, we can pick a positive $\delta_{n}<1-\left|z_{n}\right|$ such that $\left|f_{n}(z)\right| \geq 1 / 2$ whenever $\left|z-z_{n}\right|<\delta_{n}$. Because $z_{n}$ is a point of density of the set $A_{n}$, the sets $B_{n}=A_{n} \cap\left\{z \in \mathbb{C}:\left|z-z_{n}\right|<\delta_{n}\right\}$ have positive measure and the property that $\left|f_{n}(z)\right| \geq 1 / 2$ for all $z \in B_{n}$. Using that $g \in$ $H^{\infty}(\mathbb{D})_{b}$ and $f_{n} \rightarrow 0$ weakly in $H^{\infty}(\mathbb{D})$, there exist $h_{n} \in H^{\infty}(\mathbb{D})$ for which $\left\|f_{n} g-h_{n}\right\|_{\infty} \rightarrow 0$ as $n \rightarrow \infty$. As in the second paragraph of the proof this implies that $\left\|g^{*} f_{n}^{*}-h_{n}^{*}\right\|_{L^{\infty}(\mathbb{T})} \rightarrow 0$ as $n \rightarrow \infty$. Since $g^{*}=0$ almost everywhere on $\mathbb{T}$, we get $\left\|h_{n}^{*}\right\|_{L^{\infty}(\mathbb{T})} \rightarrow 0$ as $n \rightarrow \infty$, and conclude that $\left\|h_{n}\right\|_{\infty} \rightarrow 0$ as $n \rightarrow \infty$. But then it follows that $\left\|f_{n} g\right\|_{\infty} \rightarrow 0$ as $n \rightarrow \infty$. However, for $z \in B_{n}$ we 
have $\left|f_{n}(z) g(z)\right| \geq(1 / 2)(\delta / 2)=\delta / 4$, so that $\left\|f_{n} g\right\|_{\infty} \geq \delta / 4$ for all $n \in \mathbb{Z}^{+}$. This contradiction establishes our claim that $g \in V$, and hence $f=\mathscr{P}\left[f^{*}\right]+g \in H^{\infty}(\mathbb{D})+C(\overline{\mathbb{D}})+V$.

So far we have shown that $H^{\infty}(\mathbb{D})_{b}=H^{\infty}(\mathbb{D})_{N}=H^{\infty}(\mathbb{D})+C(\overline{\mathbb{D}})+$ $V$. Recalling the inclusions $H^{\infty}(\mathbb{D})_{N} \subseteq H^{\infty}(\mathbb{D})_{w c}$ and $H^{\infty}(\mathbb{D})_{w c} \subseteq$ $H^{\infty}(\mathbb{D})_{b}$ (the last inclusion because $H^{\infty}(\mathbb{D})$ has the Dunford-Pettis property) the theorem follows.

REMARK 13. Theorem 12 can be used to give an alternative proof of Theorem 10: Clearly $\omega(f, z)=0$ if $f \in H^{\infty}(\mathbb{D})+C(\overline{\mathbb{D}})$. It is easily seen that $\omega(f, z) \leq 2\left\|f \chi_{\mathbb{D} \backslash r \mathbb{D}}\right\|_{\infty}$ for $r<|z|<1$, implying that if $f \in V$, then $\omega(f, z) \rightarrow 0$ as $|z| \rightarrow 1^{-}$

5. Operator theoretic aspects. In this section we relate the Bourgain algebra of $H^{\infty}(\mathbb{D})$ to the algebra of bounded symbols for which the associated Hankel operators are compact operators on the Bergman space of the unit disk.

Let $L_{a}^{2}$ denote the Bergman space, that is, the space of square integrable analytic functions on $\mathbb{D}$. Let $d A$ denote Lebesgue area measure on $\mathbb{D}$, normalized so that $\mathbb{D}$ has measure 1 . Since $L_{a}^{2}$ is a closed subspace of $L^{2}(\mathbb{D}, d A)$ there is an orthogonal projection $P$ of $L^{2}(\mathbb{D}, d A)$ onto $L_{a}^{2}$. For $f \in L^{\infty}(\mathbb{D})$ the Hankel operator with symbol $f$, denoted by $H_{f}$, is the operator from $L_{a}^{2}$ into $L^{2}(\mathbb{D}, d A)$ defined by

$$
H_{f} g=(I-P)(f g), \quad g \in L_{a}^{2} .
$$

Clearly $H_{f}$ is a bounded operator with norm $\left\|H_{f}\right\| \leq\|f\|_{\infty}$. Let

$$
A Q=\left\{f \in L^{\infty}(\mathbb{D}): H_{f} \text { is compact }\right\} .
$$

It is known that $A Q$ is a closed subalgebra of $L^{\infty}(\mathbb{D})$ (see page 475 of [1]).

In the context of the unit circle, Hankel operators on the Hardy space $H^{2}$ are defined similarly. In [5] the Bourgain algebra $H^{\infty}(\mathbb{T})_{b}$ is shown to coincide with the algebra of bounded measurable functions for which the associated Hankel operator on the Hardy space $H^{2}$ is compact. In the context of the unit disk this is no longer true as is shown in the following theorem.

THEOREM 14. $H^{\infty}(\mathbb{D})_{b} \subsetneq A Q$.

Proof. We will first show that $H^{\infty}(\mathbb{D})_{b} \subseteq A Q$. The inclusion $H^{\infty}(\mathbb{D}) \subseteq A Q$ is trivial, for if $f \in H^{\infty}(\mathbb{D})$, then $H_{f}$ is the zero operator. Using Theorem 12 it is enough to show the inclusions 
$C(\overline{\mathbb{D}}) \subseteq A Q$ and $V \subseteq A Q$. We will use a criterion for compactness of Hankel operators obtained in K. Stroethoff [15]. For $\lambda \in \mathbb{D}$ let $\varphi_{\lambda}$ be the Möbius function defined by $\varphi_{\lambda}(z)=\frac{\lambda-z}{1-\bar{\lambda} z}, z \in \mathbb{D}$. Theorem 6 in [15], then, states that for $f \in L^{\infty}(\mathbb{D}): f \in A Q$ if and only if $\left\|f \circ \varphi_{\lambda}-P\left(f \circ \varphi_{\lambda}\right)\right\|_{2} \rightarrow 0$ as $|\lambda| \rightarrow 1^{-}$.

Now, if $f \in C(\overline{\mathbb{D}})$, then $\left|f \circ \varphi_{\lambda}(z)-f(\lambda)\right| \rightarrow 0$ for each $z \in \mathbb{D}$ as $|\lambda| \rightarrow 1^{-}$(because $\left|\varphi_{\lambda}(z)-\lambda\right|=(1-|\lambda|)|z| /|1-\bar{\lambda} z| \rightarrow 0$ as $\left.|\lambda| \rightarrow 1^{-}\right)$, and by the dominated convergence theorem, $\left\|f \circ \varphi_{\lambda}-f(\lambda)\right\|_{2} \rightarrow 0$ as $|\lambda| \rightarrow 1^{-}$. Consequently,

$$
\left\|f \circ \varphi_{\lambda}-P\left(f \circ \varphi_{\lambda}\right)\right\|_{2}=\left\|(I-P)\left(f \circ \varphi_{\lambda}-f(\lambda)\right)\right\|_{2} \rightarrow 0
$$

as $|\lambda| \rightarrow 1^{-}$, and it follows that $f \in A Q$. Thus $C(\overline{\mathbb{D}}) \subseteq A Q$.

For $0<r<1$ and $\lambda \in \mathbb{D}$ write $D(\lambda, r)=\varphi_{\lambda}(r \mathbb{D})$. Then $|D(\lambda, r)|=$ $r^{2}\left(1-|\lambda|^{2}\right)^{2} /\left(1-r^{2}|\lambda|^{2}\right)^{2}$ (see, for example, [9]). If $f \in L^{\infty}(\mathbb{D})$, then for $0<r<1$ we have

$$
\begin{aligned}
\int_{\mathbb{D}}\left|f \circ \varphi_{\lambda}\right|^{2} d A & =\int_{D(\lambda, r)}\left|f \circ \varphi_{\lambda}\right|^{2} d A+\int_{\mathbb{D} \backslash D(\lambda, r)}\left|f \circ \varphi_{\lambda}\right|^{2} d A \\
& \leq\|f\|_{\infty}^{2}|D(\lambda, r)|+\int_{\mathbb{D} \backslash r \mathbb{D}}|f(w)|^{2}\left|\varphi_{\lambda}^{\prime}(w)\right|^{2} d A(w) \\
& \leq\|f\|_{\infty}^{2}|D(\lambda, r)|+\left\|f \chi_{\mathbb{D} \backslash r \mathbb{D}}\right\|_{\infty}^{2} \int_{\mathbb{D}}\left|\varphi_{\lambda}^{\prime}(w)\right|^{2} d A(w) \\
& =\|f\|_{\infty}^{2}|D(\lambda, r)|+\| f \chi_{\mathbb{D} \backslash r \mathbb{D} \|_{\infty}^{2} .}
\end{aligned}
$$

Hence

$\lim \sup \left\|f \circ \varphi_{\lambda}\right\|_{2} \leq\left\|f \chi_{\mathbb{D} \backslash r \mathbb{D}}\right\|_{\infty}$

$$
|\lambda| \rightarrow 1^{-}
$$

So if $f \in V$, then it follows that $\left\|f \circ \varphi_{\lambda}\right\|_{2} \rightarrow 0$ as $|\lambda| \rightarrow 1^{-}$, so that $\left\|f \circ \varphi_{\lambda}-P\left(f \circ \varphi_{\lambda}\right)\right\|_{2} \rightarrow 0$ as $|\lambda| \rightarrow 1^{-}$, and therefore $f \in A Q$.

It remains to exhibit a function $f$ in $A Q$ which is not in $H^{\infty}(\mathbb{D})_{b}$. Let $f$ be as in Example 5. Then $f \notin H^{\infty}(\mathbb{D})_{b}$, and we will show that for appropriately chosen $r_{n}, f \in A Q$. We recall the following formula for $H_{f}$ (see [15]):

$$
\left(H_{f} g\right)(z)=\int_{\mathbb{D}} \frac{f(z)-f(w)}{(1-z \bar{w})^{2}} g(w) d A(w),
$$

for $g \in L_{a}^{2}, z \in \mathbb{D}$.

The operator $H_{f}$ will be Hilbert-Schmidt, and thus compact, if we make sure that

$$
\int_{\mathbb{D}} \int_{\mathbb{D}} \frac{|f(z)-f(w)|^{2}}{|1-z \bar{w}|^{4}} d A(z) d A(w)<\infty
$$


We choose $r_{2 n-1}=1-\frac{1}{n}$ and $r_{2 n}=1-\frac{1}{n+2^{-n}}$ for $n \geq 1$. Using that $f=\chi_{S}$, we have

$$
\begin{aligned}
\int_{\mathbb{D}} \int_{\mathbb{D}} & \frac{|f(z)-f(w)|^{2}}{|1-z \bar{w}|^{4}} d A(z) d A(w) \\
& \leq 2 \int_{S} \int_{\mathbb{D}} \frac{1}{|1-z \bar{w}|^{4}} d A(z) d A(w) \\
& =2 \int_{S} \frac{1}{\left(1-|w|^{2}\right)^{2}} d A(w) \leq 2 \sum_{n=1}^{\infty} \int_{r_{2 n-1}}^{r_{2 n}} \frac{2}{(1-r)^{2}} d r \\
& =4 \sum_{n=1}^{\infty}\left(\frac{1}{1-r_{2 n}}-\frac{1}{1-r_{2 n-1}}\right) \leq 4 \sum_{n=1}^{\infty} 2^{-n}=4
\end{aligned}
$$

Thus $f \in A Q$.

6. On the Bourgain algebra of the disk algebra. Let $A(\mathbb{D})_{b}$ be the Bourgain algebra of the disk algebra $A(\mathbb{D})$ relative to $L^{\infty}(\mathbb{D})$. We know that $A(\mathbb{D})_{b} \subseteq B V$ (by Remark 9) and that $A(\mathbb{D})$ contains many non-analytic functions since both $C(\overline{\mathbb{D}})$ and $V$ are subsets of $A(\mathbb{D})_{b}$. We will show that to some extent the boundary values of analytic functions in $A(\mathbb{D})_{b}$ are restricted. For this purpose we set

$$
A H=A(\mathbb{D})_{b} \cap H^{\infty}(\mathbb{D})
$$

and note that $A H$ is a Banach algebra between $A(\mathbb{D})$ and $H^{\infty}(\mathbb{D})$.

Proposition 15. If $f \in H^{\infty}(\mathbb{D})$ is continuous on $\overline{\mathbb{D}} \backslash\left\{\zeta_{1}, \ldots, \zeta_{k}\right\}$, where $\zeta_{1}, \ldots, \zeta_{k} \in \mathbb{T}$, then $f \in A H$.

Proof. There is no loss of generality in assuming $k=1, \zeta_{1}=1$ and $\|f\|_{\infty} \leq 1$. Let $\left\{f_{n}\right\}$ be a weakly null sequence in $A(\mathbb{D})$. Note that $a_{n}=\left|f_{n}(1)\right|+n^{-1} \rightarrow 0$ as $n \rightarrow \infty$. For each $n \in \mathbb{Z}^{+}$, we can choose $\delta_{n}>0$ so that $\left|f_{n}(z)\right|<2 a_{n}$ if $z \in \mathbb{D}$ and $|z-1|<2 \delta_{n}$. Fix an $n \in \mathbb{Z}^{+}$, and choose $r_{n} \in\left(1-\delta_{n}, 1\right)$ so that

$$
\left|f(z) f_{n}(z)-f\left(r_{n} z\right) f_{n}\left(r_{n} z\right)\right|<4 a_{n}
$$

whenever $z \in \mathbb{D}$ and $|z-1|>\delta_{n}$. Note that then $\left|r_{n} z-1\right| \leq$ $\left(1-r_{n}\right)|z|+|z-1|<2 \delta_{n}$ whenever $z \in \mathbb{D}$ and $|z-1|<\delta_{n}$. Choosing $g_{n}(z)=f\left(r_{n} z\right) f_{n}\left(r_{n} z\right)$ in $A(\mathbb{D})$ we have $\left\|f f_{n}-g_{n}\right\|_{\infty} \leq 4 a_{n}$.

If $f \in L^{\infty}(\mathbb{D})$ and $\zeta \in \mathbb{T}$ the essential cluster set of $f$ at $\zeta$ is

$$
\mathscr{C}(f, \zeta)=\bigcap_{0<\delta<1} \overline{R_{f}(\zeta, \delta)},
$$

where $R_{f}(\zeta, \delta)$ denotes the essential range of the function $f \mid E(\zeta, \delta)$. The diameter of a set $K \subseteq \mathbb{C}$ is written $\operatorname{diam}(K)$. 
Proposition 16. If $f \in A(\mathbb{D})_{b}$, and $Z \subseteq \mathbb{T}$ is an infinite set, then $\inf _{\zeta \in Z} \operatorname{diam} \mathscr{C}(f, \zeta)=0$.

Proof. Assume there are distinct points $\zeta_{n}$ in $\mathbb{T}$ such that

$$
\operatorname{diam} \mathscr{C}\left(f, \zeta_{n}\right) \geq \delta>0, \quad \text { for } n \in \mathbb{Z}^{+} .
$$

Then it is clear that $\omega\left(f, \zeta_{n}\right) \geq \delta>0$, for $n \in \mathbb{Z}^{+}$. By passing to a subsequence we may assume that $\zeta_{n} \rightarrow \zeta$ as $n \rightarrow \infty$. Choose $\left\{f_{n}\right\}$ in $A(\mathbb{D})$ peaking at $\zeta_{n}$ and tending to zero weakly in $A(\mathbb{D})$. Then there is a sequence $\left\{g_{n}\right\}$ in $A(\mathbb{D})$ such that $\varepsilon_{n}=\left\|f f_{n}-g_{n}\right\|_{\infty} \rightarrow 0$ as $n \rightarrow \infty$. Then for each $n \in \mathbb{Z}^{+}$we have

$$
\delta \leq \omega\left(f, \zeta_{n}\right)=\omega\left(f f_{n}, \zeta_{n}\right)=\omega\left(f f_{n}-g_{n}, \zeta_{n}\right) \leq 2 \varepsilon_{n},
$$

a contradiction.

REMARK 17 . The above proof actually shows:

If $\mathscr{A}$ is a subalgebra of $L^{\infty}(\mathbb{D})$ with $A(\mathbb{D}) \subseteq \mathscr{A} \subseteq C(\overline{\mathbb{D}})$, and if $f \in \mathscr{A}_{b}$, then $\inf _{\zeta \in Z} \operatorname{diam} \mathscr{C}(f, \zeta)=0$, for any infinite set $Z \subseteq \mathbb{T}$.

The following corollaries place restrictions on the boundary values of analytic functions in $A(\mathbb{D})_{b}$.

COROLlary 18. If $B$ is a Blaschke product whose zero set has infinitely many accumulation points, then $B \notin A H$.

Proof. If $\zeta$ is an accumulation point of the zeros of $B$, then it is easily verified that $\operatorname{diam} \mathscr{C}(B, \zeta) \geq 1$.

The above corollary implies that $A(\mathbb{D})_{b} \neq H^{\infty}(\mathbb{D})_{b}$.

COROllary 19. Let $G \in H^{\infty}(\mathbb{D})$ be nonconstant and suppose there is a set $\left\{\zeta_{k}: k \in \mathbb{Z}^{+}\right\} \subseteq \mathbb{T}$ such that $G^{*}\left(\zeta_{k}\right)=\alpha$ for all $k \in \mathbb{Z}^{+}$and $\left\{\zeta_{k}: k \in \mathbb{Z}^{+}\right\} \cap \mathbb{T}$ contains an arc. Then $G \notin A H$.

Proof. For each $\zeta$ in $\overline{\left\{\zeta_{k}: k \in \mathbb{Z}^{+}\right\}} \cap \mathbb{T}$ one has $\operatorname{diam} \mathscr{C}(G, \zeta) \geq$ $\left|G^{*}(\zeta)-\alpha\right|$.

7. Remarks and open questions. For Douglas algebras $\mathscr{A}$ and $\mathscr{B}$ a monotonicity theorem is known ([12], Theorem 4): If $H^{\infty}(\mathbb{T}) \subseteq \mathscr{A} \subseteq$ $\mathscr{B} \subseteq L^{\infty}(\mathbb{T})$, then $\mathscr{A}_{b} \subseteq \mathscr{B}_{b}$. This result fails if the Douglas algebra hypothesis is relaxed. For $\mathscr{A}=C(\mathbb{T})$ and $\mathscr{B}=H^{\infty}(\mathbb{T})_{b}$ we have $A(\mathbb{T}) \subseteq \mathscr{A} \subseteq \mathscr{B}$ and $\mathscr{A}_{b} \nsubseteq \mathscr{B}_{b}$. To see that $A_{b} \nsubseteq \mathscr{B}_{b}$ let $E \subseteq \mathbb{T}$ be a proper arc and consider the characteristic function $\chi_{E}$. It is 
straightforward to verify that $\chi_{E} \in \mathscr{A}_{b}=C(\mathbb{T})_{b}$. On the other hand $\chi_{E} \notin \mathscr{B}_{b}=H^{\infty}(\mathbb{T})_{b b}=H^{\infty}(\mathbb{T})_{b}=\mathscr{B}([12]$, Corollary 9) because the maximal ideal space of $H^{\infty}(\mathbb{T})_{b} \quad\left(=H^{\infty}(\mathbb{T})+C(\mathbb{T})\right)$ is connected and so $H^{\infty}(\mathbb{T})_{b}$ can contain no non-trivial idempotent ([8], page 188; [9], Chapter IX, Theorem 2.2). Alternatively, one can directly verify that $\chi_{E} \notin V M O$. However, if $\chi_{E} \in H^{\infty}(\mathbb{T})_{b}$, then $\chi_{E} \in V M O$ by an argument similar to Theorem 2.3 in Chapter IX of [9].

For Bourgain algebras relative to $L^{\infty}(\mathbb{D})$ monotonicity does not hold in general: $V \subseteq H^{\infty}(\mathbb{D})_{b}$, but $V_{b} \nsubseteq H^{\infty}(\mathbb{D})_{b b}$. To see that $V_{b} \nsubseteq H^{\infty}(\mathbb{D})_{b b}$ note that $V$ is an ideal in $L^{\infty}(\mathbb{D})$ so that $V_{b}=L^{\infty}(\mathbb{D})$, but $H^{\infty}(\mathbb{D})_{b b} \subseteq B V$. It would be interesting to know whether the monotonicity result of [12] holds in the $L^{\infty}(\mathbb{D})$ setting: if $\mathscr{A}$ and $\mathscr{B}$ are subalgebras of $L^{\infty}(\mathbb{D})$ with $H^{\infty}(\mathbb{D}) \subseteq \mathscr{A} \subseteq \mathscr{B}$, is it true that $\mathscr{A}_{b} \subseteq \mathscr{B}_{b}$ ?

For the polydisk $\left(X=\mathbb{D}^{n}\right.$ or $\left.X=\mathbb{T}^{n}\right)$ the Bourgain algebras $A(X)_{b}=A(X)$ and $H^{\infty}(X)_{b}=H^{\infty}(X)$, have been determined by J. Cima and W. Wogen and also by K. Izuchi [13]. On the ball $\mathbb{B}^{n}$ the result $A\left(\partial \mathbb{B}^{n}\right)_{b}=C\left(\partial \mathbb{B}^{n}\right)$ (relative to $C\left(\partial \mathbb{B}^{n}\right)$ ) is implicit in $\mathbf{J}$. Bourgain [3]. Concerning $H^{\infty}\left(\partial \mathbb{B}^{n}\right)_{b}$, a theorem of W. Rudin [14] says that $H^{\infty}\left(\partial \mathbb{B}^{n}\right)+C\left(\partial \mathbb{B}^{n}\right)$ is a closed subalgebra of $L^{\infty}\left(\partial \mathbb{B}^{n}\right)$. K. Izuchi [13] has recently shown that $H^{\infty}\left(\partial \mathbb{B}^{n}\right)+C\left(\partial \mathbb{B}^{n}\right)=H^{\infty}\left(\partial \mathbb{B}^{n}\right)_{b}$ (relative to $L^{\infty}\left(\partial \mathbb{B}^{n}\right)$ ) .

\section{REFERENCES}

[1] S. Axler, J. B. Conway and G. McDonald, Toeplitz operators on Bergman spaces, Canad. J. Math., 34 (1982), 466-483.

[2] J. Bourgain, New Banach space properties of the disc algebra and $H^{\infty}$, Acta Math., 152 (1984), 1-48.

[3] _ The Dunford-Pettis property for the ball algebras, the polydisc-algebra and the Sobolev spaces, Studia Math., 77 (1984), 245-253.

[4] S.-Y. Chang and D. E. Marshall, Some algebras of bounded analytic functions containing the disc algebra, pp. 12-20 in Banach Spaces of Analytic Functions, Lecture Notes in Math., Vol. 604, Springer Verlag, Berlin and New York, 1977.

[5] J. Cima, S. Janson and K. Yale, Completely continuous Hankel operators on $H^{\infty}$ and Bourgain algebras, Proc. Amer. Math. Soc., 105 (1989), 121-125.

[6] J. Cima and R. Timoney, The Dunford-Pettis property for certain planar uniform algebras, Michigan Math. J., 34 (1987), 99-104.

[7] B. Cole and T. W. Gamelin, Tight uniform algebras, J. Funct. Anal., 46 (1982), 158-220.

[8] K. Hoffman, Banach Spaces of Analytic Functions, Prentice-Hall, Englewood Cliffs, New Jersey, 1962.

[9] J. Garnett, Bounded Analytic Functions, Academic Press, New York, 1981.

[10] P. Ghatage, S. Sun and D. Zheng, A remark on Bourgain algebras on the disk, Proc. Amer. Math. Soc., 114 (1992), 395-398. 
[11] P. Gorkin, Algebras of bounded functions on the disk, pp. 155-167 in "Proceedings Conference on Function Spaces" (edited by K. Jarosz), Lecture Notes in Pure and Appl. Math., Vol. 136, Marcel Dekker, 1992.

[12] P. Gorkin, K. Izuchi and R. Mortini, Bourgain algebras of Douglas algebras, Canad. J. Math., 44 (1992), 797-804.

[13] K. Izuchi, Bourgain algebras of the disk, polydisk and ball algebras, Duke Math. J., 66 (1992), 503-519.

[14] W. Rudin, Spaces of the type $H^{\infty}+C$, Ann. Inst. Fourier Grenoble, 25 (1975), 99-125.

[15] K. Stroethoff, Compact Hankel operators on the Bergman space, Illinois J. Math., 34 (1990), 159-174.

[16] K. Yale, Bourgain algebras, pp. 413-422 in "Proceedings Conference on Function Spaces" (edited by K. Jarosz), Lecture Notes in Pure and Appl. Math., Vol. 136, Marcel Dekker, 1992.

[17] D. Zheng, Bourgain algebras of some algebras on the disk, preprint.

Received February 15, 1991 and in revised form April 20, 1992. The first author appreciated the hospitality of the University of Montana while this work was done; the second author acknowledges partial support of grants from the Montana University System and the University of Montana.

UNiversity OF NORTH CAROLINA

Chapel Hill, NC 27514

AND

UNIVERSITY OF MONTANA

Missoula, MT 59812 



\section{CONTENTS}

G. D. Anderson, M. K. Vamanamurthy, and M. Vuorinen, Inequalities for quasi-

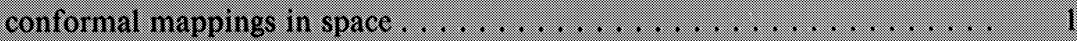

T. Bhattacharya, A nonexistence result for the $n$-Laplacian . . . . . . . . . . . . 19

J. A. Cima, K. Stroethoff, and K. Yale, Bourgain algebras on the unit disk . . . . . 27

J. A. Fridy and C. Orhan, Lacunary statistical convergence . . . . . . . . . . . . . . 43

D. Grenier, On the shape of fundamental domains in $\mathrm{GL}(n, \mathbf{R}) / \mathrm{O}(n) \ldots \ldots . . .53$

B. Jiang and J. Guo, Fixed points of surface diffeomorphisms . . . . . . . . . . . 67

P. Lejarraga, The moduli of rational Weierstrass fibrations over $\mathbf{P}^{\prime}$ : singularities 91

G. J. Martin, On discrete isometry groups of negative curvature . . . . . . . . . . 109

T. Nakashima, Adjoint linear systems on a surface of general type in positive characteristic . . . . . . . . . . . . . . . . . . . . . . . . . 129

B. Ralph, A homotopy transfer for finite group actions $\ldots \ldots \ldots \ldots \ldots \ldots \ldots$

Y. Rong, Maps between Seifert fibered spaces of infinite $\pi_{1} \ldots \ldots \ldots \ldots \ldots, 143$

J.-Y. Shi, Some numeric results on root systems . . . . . . . . . . . . 155

E. Spanier, Singular homology and cohomology with local coefficients and duality

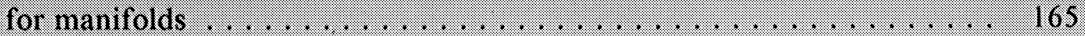




\section{PACIFIC JOURNAL OF MATHEMATICS}

Volume $160 \quad$ No. $1 \quad$ September 1993

Inequalities for quasiconformal mappings in space

GLEN DOUglas ANDERson, MaVina KRISHNa VAMANAMURThy and MATTI VUORINEN

A nonexistence result for the $n$-Laplacian

TILAK BHATTACHARYA

Bourgain algebras on the unit disk

Joseph A. Cima, Karel M. Stroethoff and Keith Yale

Lacunary statistical convergence

JOHN ALBERT FRIDY and CIHAN ORHAN

On the shape of fundamental domains in $\operatorname{GL}(n, \mathbf{R}) / \mathrm{O}(n)$

DOUGLAS MARTIN GRENIER

Fixed points of surface diffeomorphisms

BOJU JIANG and JIANHAN GUO

The moduli of rational Weierstrass fibrations over $\mathbf{P}^{1}$ : singularities

PABLO LEJARRAGA

On discrete isometry groups of negative curvature

GAVEN MARTIN

Adjoint linear systems on a surface of general type in positive characteristic

TOHRU NAKASHIMA

A homotopy transfer for finite group actions

WILLIAM J. RALPH

Maps between Seifert fibered spaces of infinite $\pi_{1}$

YONGWU RONG

Some numeric results on root systems

J. Y. SHI

Singular homology and cohomology with local coefficients and duality for manifolds

EDWIN SPANIER 